\title{
The Origin of the High-Energy Activity at the Galactic Center
}

\author{
Farhad Yusef-Zadeh*, William Purcell* \& Eric Gotthelf ${ }^{\dagger}$ \\ *Dept. Physics and Astronomy, Northwestern University, Evanston, Ill. 60208 \\ ${ }^{\dagger} N A S A / G S F C$, Greenbelt, MD 20771
}

\begin{abstract}
Recent X-ray and gamma-ray observations of the Galactic center region by the ASCA and CGRO/EGRET instruments show evidence of 2-10 keV and > 1 $\mathrm{GeV}$ continuum emission as well as 6.7 and $6.4 \mathrm{keV}$ line emission from the inner $0.2^{\circ}$ of the Galactic center. This region is also known to host a bright nonthermal radio continuum source Sgr A East and a dense molecular cloud M-0.02-0.07 known as the $50 \mathrm{~km} \mathrm{~s}^{-1}$ cloud. The oval-shaped nonthermal Sgr A East is physically interacting with M-0.02-0.07 at the Galactic center. A comparison between the distribution of ionized, synchrotron and neutral gas suggests a self-consistent interpretation of the high-energy activity at the Galactic center. Our preliminary analysis of the data suggest a shock model of cosmic ray acceleration at the site of the interaction to explain the enhanced $\mathrm{GeV} \gamma$-ray emission. We also address a number of issues related to the spatial correlation of the diffuse radio and X-ray emitting gas as well as to the origin of the fluorescent 6.4 and $6.7 \mathrm{keV}$ emission at the Galactic center.
\end{abstract}

\section{INTRODUCTION}

Radio View of the Galactic Center Radio continuum observations of the inner $15^{\prime}$ of the Galactic center show two prominent radio continuum structures known as the Sgr A complex and the filamentary continuum Arc. The Sgr A Complex consists of Sgr A East and its halo as well as Sgr A* and its thermal orbiting gas Sgr A West. Sgr $\mathrm{A}^{*}$ is unique and considered by many to be a massive black hole with a mass of $10^{6} \mathrm{M}_{\odot}$ at the Galactic center. The oval-shaped structure known as Sgr A East is thought to be the remnant of an explosion located just behind the Galactic center (Yusef-Zadeh and Morris 1987; Pedlar et al. 1989), A number of authors (Khokhlov \& Melia 1996; Mezger et al. 1989; Yusef-Zadeh and Morris 1987) question the interpretation of Sgr A East as a standard SNR. Khokhlov \& Melia (1996) have considered that Sgr A East is the remnant of star that is tidally disrupted by a massive black hole, presumably by Sgr A*. The explosion energy is estimated to be an order of magnitude more than the energy released by a typical supernova. There is also considerable evidence that this explosion occurred inside the dense molecular cloud M-0.02-0.07, thus depositing more than $4 \times 10^{52}$ ergs in the ISM (Mezger et al. 1989). Recent discovery of OH(1720MHz) masers at the interface of the $50 \mathrm{~km} \mathrm{~s}^{-1}$ molecular cloud and Sgr A East showed conclusively that these two are physically interacting with each other (Yusef-Zadeh et al. 1996).

On the largest scale, there is a diffuse $7-10^{\prime}$ halo of nonthermal continuum emission surrounding the oval-shaped radio structure Sgr A East. The spectrum of the halo tends to be steeper than Sgr A East and is primarily nonthermal with the 
energy spectral index of $>3$. The optical depth toward Sgr A East and the halo at low frequencies lead Pedlar et al. (1989) to consider a mixture of both thermal and nonthermal gas but displaced to the front side of Sgr A East.

The Arc is a nonthermal filamentary source located near $1 \approx 0.18^{0}$ and runs in the direction perpendicular to the Galactic plane. The filaments are linearly polarized showing evidence that they are tracing magnetic field lines and emitting synchrotron radiation. The spectrum of the filaments is unusual in that it is flatter than typical nonthermal features. The energy spectrum of relativistic particles have a spectral index of $\approx 1.6$ in the radio wavelengths. A number of Galactic center molecular clouds appear to outline the linear filaments, prompting the hypothesis that the filaments and clouds are physically interacting with each other, in which case the field strength is estimated to be at least $1 \mathrm{mG}$ in order for the filaments to resist deflection at points of interaction with the clouds (Yusef-Zadeh and Morris 1987; Serabyn and Morris 1994).

X-Ray View of the Galactic Center Recent ASCA observations of the Galactic center showed conclusively the evidence for diffuse X-ray emission arising from the inner $15^{\prime}$ of the Galactic center (Koyama et al. 1996). The continuum radiation is accounted for by thermal plasma having temperature of $10 \mathrm{keV}$. The strongest continuum radiation from the inner $30^{\prime}$ in the energy band between 0.7 and $10 \mathrm{keV}$ arises from within the shell of Sgr A East and is somewhat elongated along the Galactic plane. The electron density and the thermal energy of thermal gas within the shell of Sgr A East are estimated to be $6 \mathrm{~cm}^{-3}$ and $3 \times 10^{50} \mathrm{ergs}$, respectively (Koyama et al. 1996). Weak and diffuse emission is also seen corresponding to the radio halo as well along the Galactic plane both in the positive and negative longitudes, but with low surface brightness. This weak emission extends over as far as $80 \mathrm{pc}\left(33^{\prime}\right)$ on either side of the Galactic center. The strongest diffuse emission beyond the Sgr A complex arises from the positive longitude side outlined by the nonthermal radio filaments of the Arc. Figure 1 shows X-ray contours superimposed on the radio image displaying the Sgr A East shell, its halo and the filaments in the Arc. The electron density and the thermal energy of the hot gas outside the Sgr A complex are estimated to be about $0.3-0.4 \mathrm{~cm}^{-3}$ and $0.5-1 \times 10^{53}$ ergs, respectively (Koyama et al. 1996).

One of the more fascinating aspect of ASCA observations of the Galactic center is the evidence of $6.4 \mathrm{keV}$ emission peaking on two molecular clouds in the region between the Arc and the Sgr A complex and in Sgr B2. This fluorescent K $\alpha$ line emission results from the K-shell photoionization of iron atoms.

Gamma-Ray View of the Galactic Center Recent report of high energy (30 MeV - $30 \mathrm{GeV}$ ) continuum emission from the Galactic center based on EGRET observations (Mattox 1997) indicate a source with a luminosity of $5 \times 10^{36} \mathrm{erg} \mathrm{s}^{-1}$ at the distance of the galactic center. The source is situated within $0.2^{0}$ of the Galactic center and could be either compact or diffuse within 100 pc of the Galactic center. The energy spectrum of this source is fit by a power law having an index of 1.7 which is harder than typical EGRET sources in the Galactic plane. This source 
has been considered to be associated with the Arc (Pohl 1997), or with Sgr A*, or possibly to have a diffuse origin (Thompson et al. 1996; Mattox 1997).

\section{DISCUSSION}

Sgr A East as the Source of High-Energy Activity The hypothesis that we are considering involves Sgr A East, the most energetic source in the Galactic center region. This source has been considered to be due to an unusual explosion, perhaps a Seyfert-like activity as seen in the nucleus of spiral galaxies (Pedlar et al. 1989). A number of observations indicate that Sgr A East is unusual and more energetic than a typical supernova, having released more than $5 \times 10^{52}$ ergs into the Galactic center region (e.g. Mezger et al. 1989).

Since the interaction of Sgr A East with the $50 \mathrm{~km} \mathrm{~s}^{-1}$ molecular cloud is well established, the high-energy cosmic rays responsible for radio, X-ray and $\gamma$-ray emission could be generated at the site of the interaction of Sgr A East and its molecular cloud before diffusing out along the Galactic plane. In this hypothesis, the EGRET source is considered to be diffuse and the $\gamma$-ray spectrum is due to accelerated cosmic rays at the site of the interaction of the explosive event with the giant molecular cloud. The western edge of the $50 \mathrm{~km} \mathrm{~s}^{-1}$ giant molecular cloud is interacting with the Sgr A East shell whereas the eastern edge of the cloud appears to be outlined by the nonthermal linear filaments that cross the Galactic plane near $l \approx 0.2^{0}$. Figure 2 shows the overall distribution of ${ }^{13} \mathrm{CO}$ gas between 30 and $50 \mathrm{~km} \mathrm{~s}^{-1}$ (Bally et al. 1988) which appears to be correlated with the hot $\mathrm{X}$-ray emitting gas displayed as contours in Figure 1. In addition, the distribution of $6.4 \mathrm{keV}$ emission from the inner $30^{\prime}$ of the Galactic center, as discussed below, appears to coincide with the peaks of two molecular gas clouds (Koyama et al. 1996) in M-0.02-0.07. These morphological correlations strongly suggest that Sgr A East, the $50 \mathrm{~km} \mathrm{~s}^{-1}$ molecular cloud, the hot X-ray emitting gas and the $6.4 \mathrm{keV}$ emission are physically associated implying that Sgr A East is responsible for the high-energy activity at the Galactic center.

There is considerable evidence that nonthermal particles at high energies produce synchrotron emission from Sgr A East and the Arc as well as the continuum EGRET $\gamma$-ray source (2EGJ1746-2852) seen at the Galactic center. There is also conclusive evidence that shocks exit at the interface of the $50 \mathrm{~km} \mathrm{~s}^{-1}$ molecular cloud and Sgr A East. Thus, it is plausible that the low-energy cosmic ray particles must exist from extrapolation of high-energy nonthermal particles. In fact some of the brightest supernova remnants interacting with molecular clouds and showing $\mathrm{OH}$ maser shocks at $1720 \mathrm{MHz}$ appear to have EGRET counterparts (Esposito et al. 1996).

The eastern edge of the Sgr A East lies a high density molecular core with a density of $1-2 \times 10^{6} \mathrm{~cm}^{-3}$ and a string of HII regions excited by massive stars (e.g. Serabyn et al. 1992). Both these features are associated with sites of star formation in the $50 \mathrm{~km} \mathrm{~s}^{-1}$ cloud. The cluster of hot stars and the cavity of ionized gas surrounded by the circumnuclear ring at the Galactic center begs the question 
of the association of these features with another site of star formation within the 50 $\mathrm{km} \mathrm{s}^{-1}$ cloud. We sketch a scenario in which the cluster of hot stars IRS 16 and the circumnuclear ring of molecular gas are remnants of an episode of star formation that took place in the $50 \mathrm{~km} \mathrm{~s}^{-1}$ cloud. In this picture, the stars and the gas are captured at the Galactic center as the giant molecular cloud passed the Galactic center and is presently behind Sgr A West.

The Nature of $10^{7}-10^{8} \mathrm{~K}$ Emission The $50 \mathrm{~km} \mathrm{~s}^{-1}$ molecular is distributed along the positive longitude side of the plane but immediately behind the Galactic center. The front side of the cloud is assumed to face Sgr A* and the shell of Sgr A East outlines the compressed molecular gas as a result of the shock wave driving into the the dense medium of M-0.02-0.07. The halo of Sgr A East seen both in the radio and X-ray wavelengths is a secondary manifestation of the explosion. The hot gas and the accelerated cosmic rays that are produced at the site of the shock leak preferentially toward the Galactic center because Sgr A East is bounded by the giant molecular cloud. The X-ray emitting gas is most concentrated within the shell of Sgr A East resembling the structure of composite supernova remnants. A strong morphological correlation between the nonthermal radio features, the Arc and Sgr A East and its halo, and the hot and cold thermal emitting gas as noted in contours of Figures 1 and 2 indicate strongly that the X-ray gas has a nonthermal component and is mixed with thermal gas at a temperature of $10 \mathrm{keV}$. In fact, there is evidence of a hard tail in the diffuse X-ray spectrum of the inner region of the Galaxy based on Ginga observations (Yamasaki et al. 1996).

The hot plasma at this temperature can not be confined by the gravitational potential in the Galactic center, thus an alternative mechanism is required to constrain the hot gas in the region. The morphology of X-ray and radio emitting gas suggests that the X-ray gas follows the magnetized filaments before reaching the Arc. Under the assumption that the magnetic pressure of the Arc is strong enough to confine much of the hot plasma, the estimated field strength has to be greater than $0.5 \mathrm{mG}$ for an electron density of $0.3 \mathrm{~cm}^{-3}$ and a temperature of $10 \mathrm{keV}$.

The Nature of the $6.4 \mathrm{keV}$ Emission Koyama et al. (1996) argue that the hot plasma can not account for exciting the $6.4 \mathrm{keV}$ line emission since the observed Xray luminosity is an order of magnitude less than the observed $6.4 \mathrm{keV}$ line emission. Recent theoretical work by Borkowski and Szymkowiak (1997), however show that thermal electrons with few to $10 \mathrm{keV}$ energies can penetrate interstellar dust grains and produce fluorescent $\mathrm{K} \alpha$ emission through the $\mathrm{K}$ shell ionization. The advantage of this mechanism over the excitation of $\mathrm{K} \alpha$ of $\mathrm{Fe}$ in the gas phase is that the dust grains are abundant in the content of their heavy atoms. These authors consider a non-equilibrium ionization model to account for X-ray spectra of hot plasma mixed in with neutral atoms of heavy elements. The ionization parameter $\tau$ is the product of electron density $\mathrm{n}_{e}$ and time t. In the case when $\tau$ is less than $10^{13} \mathrm{~cm}^{-3} \mathrm{~s}$ all ions are in equilibrium and the contribution of $\mathrm{Fe} \mathrm{K} \alpha$ emission from dust grains is minimal. However, for the case when the plasma is underionized before the final equilibrium state, the Fe K $\alpha$ emission could be totally due to dust grains. Since 
the observed $6.4 \mathrm{keV}$ and $6.7 \mathrm{keV}$ line intensities arise from the dense molecular clouds outside the Sgr A East complex, it is plausible that the thermal electrons of the plasma with a $10 \mathrm{keV}$ temperature is exciting the $\mathrm{Fe} \mathrm{K} \alpha$ of dust grains in selected clouds where the non-equilibrium ionization model is applicable.

We believe that the two prominent clouds toward Sgr A East and Sgr A West (the circumnuclear disk) at the Galactic center, namely the $50 \mathrm{~km} \mathrm{~s}^{-1}$ and the circumnuclear neutral gas orbiting Sgr $\mathrm{A}^{*}$, do not show any $6.4 \mathrm{keV}$ line emission because the ionization parameter is close to $10^{13} \mathrm{~cm}^{-3} \mathrm{~s}$. With the expansion time scale of $5 \times 10^{4}$ yrs over 80 pcs and the electron density of $6 \mathrm{~cm}^{-3}$ in Sgr A East, $\tau=10^{-13} \mathrm{~cm}^{-3}$. However, the region where the $\mathrm{Fe} \mathrm{K} \alpha$ is seen, the electron density of the hot plasma is much lower than $6 \mathrm{~cm}^{-3}$ and therefore the non-equilibrium ionization model is applicable.

Ginga results have shown a hard tail in the spectrum of the inner degree of the Galactic center (Yamauchi et al. 1990). The hard tail beyond $10 \mathrm{keV}$ can be important in explaining the nature of $6.4 \mathrm{keV}$ emission and the strong correlation seen between $\mathrm{CO}$ and 2-10 keV spatial distributions and in determining whether the low-energy cosmic ray electrons are responsible for heating of molecular clouds near the Galactic center.

Summary A number of different observations indicate strongly the co-existence of nonthermal and thermal gas in the inner $50 \mathrm{pc}$ of the Galaxy. This mixture of gas can be accounted for in a self-consistent fashion by assuming that the center of the high-energy activity is due to the unusual explosion of Sgr A East which is expanding inside the $50 \mathrm{~km} \mathrm{~s}^{-1}$ giant molecular cloud. In this picture, the highenergy $(\approx \mathrm{GeV})$ cosmic rays accelerated by the explosion are responsible for the $\gamma$-ray emission as detected by CGRO/EGRET, the nonthermal radio continuum emission from Sgr A East and its halo, and for illumination of the magnetic field lines of the Arc. On the other hand, the low-energy $(<1 \mathrm{MeV})$ cosmic-ray particles are considered to heat the giant $50 \mathrm{~km} \mathrm{~s}^{-1}$ molecular cloud and produce the observed diffuse X-ray emission, the $6.4 \mathrm{keV}$ and the hard tail observed in the Ginga spectrum. The strong X-ray emission arising simultaneously from the Sgr A East shell and from the interior of the shell shows a resemblance to the centrally peaked morphology of composite SNR's such as W44 (e.g. Rho et al. 1994). A more detailed account of this model will be given elsewhere.

\section{REFERENCES}

1. Bally, J., et al., ApJ, 324, 223 (1988).

2. Borkowski, K. \& Szymkowiak, A.E., ApJ, 477, L49 (1997).

3. Esposito, J. A., et al., ApJ, 461, 820 (1996).

4. Khokhlov, A. \& Melia, F., ApJ, 457. L61 (1995).

5. Koyama K., et al., PASJ, 48, 249 (1996).

6. Mattox, J. R., GCNEWS, vol. 4, (Feb. 1997).

7. Mezger, P. G., et al., A.A., 209, 337 (1989).

8. Pedlar, A., et al., ApJ, 342, 769 (1989). 
FIGURE 1. Radiograph of the $\lambda 20 \mathrm{~cm}$ continuum image superimposed on X-ray contours in the energy range between 0.7 and $10 \mathrm{keV}$ with a resolution of 1'. The ASCA data was kindly provided by Dr. Koyama and Dr. Maeda.

FIGURE 2. Using the 1950 coordinates, this figure is simialr to Figure 1 except that the contours represent the distribution of ${ }^{13} \mathrm{CO}$ emission in the velocity range between 30 and $50 \mathrm{~km}$ $\mathrm{s}^{-1}$ having similar resolution to that of X-ray data shown in Figure 1 (Yusef-Zadeh 1986; Bally et al. 1988).

9. Pohl, M., A.A. 317, 441 (1997).

10. Rho, J. et al. ApJ 430, 757 (1994).

11. Serabyn, E., et al., ApJ, 395, 166 (1992).

12. Serabyn, E. \& Morris, M., ApJ, 424, L91 (1994).

13. Thompson, D.J. et al., ApJS, 207, 227 (1996).

14. Yamasaki, N.Y. et al., A.A.S, 120, 393 (1996).

15. Yamauchi, S., et al., ApJ, 365, 532 (1990).

16. Yusef-Zadeh, F. Ph.D. thesis, Columbia University (1986).

17. Yusef-Zadeh, F., et al., ApJ, 466, L25 (1996).

18. Yusef-Zadeh, F. \& Morris, M., ApJ, 320, 545 (1987). 